\title{
¿Madres? alrededor del trono
}

\author{
Games of Thrones | Thomas McCarthy y otros | 2011
}

\author{
Alejandro Willington* \\ EOL Sección Córdoba
}

Recibido: 20 de junio 2017; aceptado: 30 de junio 2017

\begin{abstract}
Resumen
El capítulo piloto de la serie televisiva Games of Thrones nos presenta tres personajes femeninos diferentes respecto de sus posiciones subjetivas. El presente trabajo los extrae para ubicar desde una lectura psicoanalítica algunas complejidades en las posiciones maternas que esta teoría plantea a partir de la experiencia clínica. Así, se desarrollan los rasgos de cada una de las tres protagonistas de la serie, tomando como punto de partida la pregunta psicoanalítica sobre la maternidad y la feminidad, anticipando desde el inicio que una posición no remite necesariamente a la otra. Se plantea así, desde el punto de vista lacaniano, que la madre es aquella parte de la sexualidad femenina que se ordena según la referencia fálica. Para Lacan, el niño en la relación dual con la madre le da lo que le falta al sujeto masculino: el objeto mismo de su existencia, apareciendo en lo real. Asimismo, el texto ubica un rasgo fundamental en estas tres madres y es la búsqueda de venganza. Desde esta perspectiva, el trabajo se orienta nuevamente en la referencia lacaniana sobre Medea, la tragedia de Eurípides, aquella mujer que mata a sus propios hijos empujada por su sed de venganza. La venganza en la mujer puede ser ilimitada, como el propio goce femenino, cuando se siente despojada, o traicionada, al precio, como señala Lacan, de ser otra que sí misma en el propio acto que consuma dicha venganza.
\end{abstract}

Palabras clave: Venganza | Mujer | Fantasma | Maternidad

Mothers? Around the throne

\begin{abstract}
The pilot chapter of the television series Games of Thrones presents us with three different female characters regarding their subjective positions. The present work extracts them to locate from a psychoanalytic reading some complexities in the maternal positions that this theory poses from the clinical experience. Thus, the traits of each of the three protagonists of the series are developed, taking as a starting point the psychoanalytic question about motherhood and femininity, anticipating from the beginning that one position does not necessarily refer to the other. Thus, from the Lacanian point of view, the mother is that part of female sexuality that is ordered according to the phallic reference. For Lacan, the child in the dual relationship with the mother gives him what the male subject lacks: the very object of his existence, appearing in the real. Also, the text places a fundamental trait in these three mothers and is the search for revenge. From this perspective, the work is again oriented to the Lacanian reference on Medea, the tragedy of Euripides, that woman who kills her own children driven by her thirst for revenge. Revenge on women can be unlimited, like female enjoyment itself, when it feels deprived or betrayed, at the price, as Lacan points out, of being other than itself in the very act that consumes such revenge.
\end{abstract}

Keywords: Revenge | Women | Ghost | Motherhood

Game of thrones, ya de entrada, pareciera decirnos que madre no hay una sola. En el relato las podemos repartir por tres: Catelyn, Daenerys y Cersei. Comencemos por Lady Stark, una mujer de familia, fuerte, ocupando su lugar de esposa y madre con temple, preocupada siempre tanto por sus hijos como por su esposo Ned Stark, Señor de Invernalia. Así es presentada al menos al comienzo de la serie, sin embargo, con el desarrollo de la misma van apareciendo otros trazos de su personalidad: su impulsividad provocará diversos contratiempos.

“Tras su asesinato y posterior resurrección como Lady Corazón de Piedra (acontecimiento que al menos hasta aquí no tuvo lugar en la serie televisiva, lo debemos rastrear en los libros o en la infinidad de plataformas online dedicadas al Juego de Tronos), el cambio será tanto físico como en la personalidad: será una mujer fría, inmisericorde y consumida por el deseo de venganza contra los enemigos de su familia” (Catelyn Tully, Wikipedia).

Daenerys Targaryen es uno de los personajes que más va mutando con el devenir de la saga, comienza siendo casi una niña, de aspecto adolescente y candoroso, es prontamente entregada por el hermano a un bárbaro, que de ese modo la transforma violentamente en mujer. Un paulatino aprendizaje de sus deberes como reina, y mujer, le van permitiendo tomar su cargo como Khaleesi de los Do-

*ale.willington@gmail.com 
thraki y así va superando penalidades. "Daenerys adquiere una personalidad mucho más decidida, audaz y valerosa" (Daenerys Targaryen, Wikipedia). Madre de dragones, esos falos llameantes serán el instrumento de venganza de sus penurias, y de las de su padre, despojado del trono de hierro. Finalmente, Cersei Lannister, la esposa del rey Robert Baratheon, presenta un carácter ególatra y sumamente ambicioso, nada la detiene en su afán de cumplir sus objetivos: "ella misma admite no amar a ninguna otra persona que no sea ella o sus hijos, pues cree que el amor hace a las personas débiles" (Cersei Lannister, Wikipedia). Su ambición más allá de todo límite o ley la empujan hasta las relaciones incestuosas con su hermano mellizo. La historia de Lucrezia Borgia se nos presenta rápidamente en el horizonte referencial de este personaje. La sed de ambición y de venganza en Cersei no tiene ningún límite, la saga de los Borgia se infiltran decididamente en Juego de Tronos a través de la dinastía Lannister.

Entonces, tres madres y un rasgo fundamental, la venganza. ¿Tres madres o tres mujeres? ¿Qué tiene para decirnos el psicoanálisis al respecto?

Nos referimos tanto a la separación entre la posición materna y la posición femenina, como a la venganza femenina.

Digamos, primero, que el saber moderno, en su vertiente médico-higieniesta o psicologista, ha intentado definir siempre a la mujer por la maternidad. Freud mismo no logró desprenderse del todo de este prejuicio. Recordemos cómo situaba en diversos textos a la mujer, definiendo su deseo por la estricta equivalencia del niño y del falo (Freud, 1916-1938 [1945]). Aunque por otro lado debamos decir que en el texto freudiano se trata de una maternidad despegada del sustrato biológico:

“Ahora bien, la pulsión, el deseo y la ley producen una «desnaturalización» de la madre misma y de la relación madre - niño: no hay madre que no sea desnaturalizada. (...) Se creía haber humanizado la mujer con la madre, pero es en el corazón de la madre que Freud hace resurgir la subversión del deseo" (Brousse, 1993, p. 30-33).

Daenerys, madre de dragones por elección y adopción, nos demuestra que la maternidad se desprende en el ser hablante del sustrato biológico de su cuerpo, elección que el personaje asume tras la pérdida de su propio embarazo y de su hombre, Khal Drogo, líder de la estirpe guerrera y bárbara de los Dothraki. Se esbozan, en este momento crucial de la trama, ciertas líneas de fuerza en la construcción del personaje, que harán de esta madre una guerrera incansable, custodiada luego por sus espadas aladas, los dragones. Cersei Lannister, en cambio, ya es presentada en el capítulo piloto como esa mujer que empuja su capricho más allá de toda ley, rechazando aquella que funda, a decir de Freud, la cultura misma. Nos referimos a la prohibición del incesto. Para que haya incesto debe haber una ley que lo sancione y borrarla de plano se pagará, como lo atestigua este potente personaje, quizás más femenino que materno, con un aire de locura.

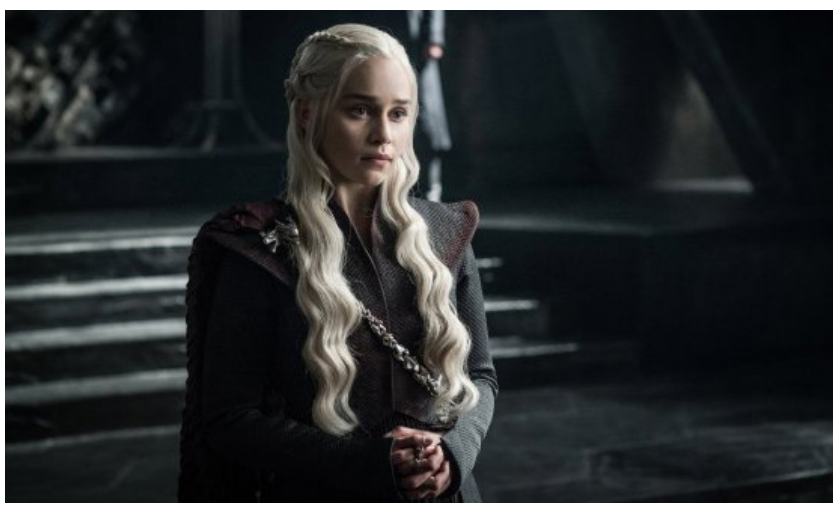

$\mathrm{Si}$ pasamos del marco freudiano al lacaniano, podemos adelantar la idea que lo que sabemos de la madre es aquella parte de la sexualidad femenina que se ordena según la referencia fálica (Brousse, 1993, p. 3033). Daenerys nos da el ejemplo más claro en la lógica de esta serie, cuando resigna su maternidad humanizada, tras haber sido despojada del niño que llevaba en su vientre. Transformándose así, tras el rito de fuego, en la guerrera que monta sus dragones "según la misma lógica del odio y la reivindicación, ... de la envidia del pene (...). La sexualidad femenina, y Lacan no dice lo contrario, es de entrada una sexualidad bajo la dominación del falo" (Brousse, 1993, p. 30-33).

Este personaje, cándido al inicio, advenido luego una valquiria con promesa de reina (eso es al menos lo que se sugiere para la última temporada que se avecina) muestra muy bien que "la maternidad es entonces una posición sexual que consagra en un sujeto femenino el sacrificio de goce implicado por la castración" (Brousse, 1993, p. 30-33). Los tres dragones con que se arma Daenerys llevarán el nombre de los tres objetos fundamentales que le fueron arrebatados: Viserion, Rhaegal y Drogon.

Ahora bien, vayamos a la tercera madre, a Catelyn, quien de entrada se presenta como la madre más abnegada, podríamos decir, hasta la más regulada. Una buena madre y una buena mujer, dedicada a sus hijos y esposo, es lo que observamos en el capítulo piloto. Pues bien, no es así como se desarrolla y termina el personaje, según leemos en el relato original, aunque aún no haya ingresado a la serie televisiva. Gradualmente la pérdida de esa especie de familia ideal que se nos dibuja al comienzo la 
va sumiendo en el odio y la venganza, para terminar con una Catelyn cuya apariencia resulta aterradora, nos referimos a la reencarnación de ella en la mujer de corazón de piedra (tras la madre, la reencarnación en la mujer, o la mujer como la verdad detrás de la madre). Esa cuya piel "se ha vuelto blanquecina, su pelo canoso y al haberle cortado el cuello apenas puede hablar de forma audible, además se deja consumir completamente por su deseo de venganza" (Catelyn Tully, Wikipedia).

En este primer capítulo vemos cómo casi pierde a su niño más pequeño, loca de dolor luego asistirá a la muerte de otros. Entonces, ¿qué son para ella estos hijos? Son los objetos que dirigen y condensan su goce, como nos dice Lacan. Porque el niño satura la falta con este objeto según la modalidad que especifica el deseo de la madre (Lacan, 2001 [2012]), sea cual sea la estructura en particular: represión, denegación o forclusión. Lacan prosigue: "Resumiendo, el niño en la relación dual con la madre le da, inmediatamente accesible, lo que le falta al sujeto masculino: el objeto mismo de su existencia, apareciendo en lo real" (Lacan, 2001 [2012], p. 394).

Esa imagen patética con la que se nos describe aquello en lo que se trasforma esa noble madre que pudimos ver en el primer capítulo, es la representación misma del desecho, casi mudo, al que queda reducida, aspirada completamente por una sed de venganza insaciable. Al ser despojada de sus niños - objetos, queda finalmente transformada en el desecho mismo de esa pérdida; es así que observamos como "la figura de la madre buena oculta el rasgo de perversión de una relación con el objeto. Así, detrás de la madre de la frustración y de la gratificación, es el puñal de Medea el que se perfila” (Brousse, 1993, 30-33).

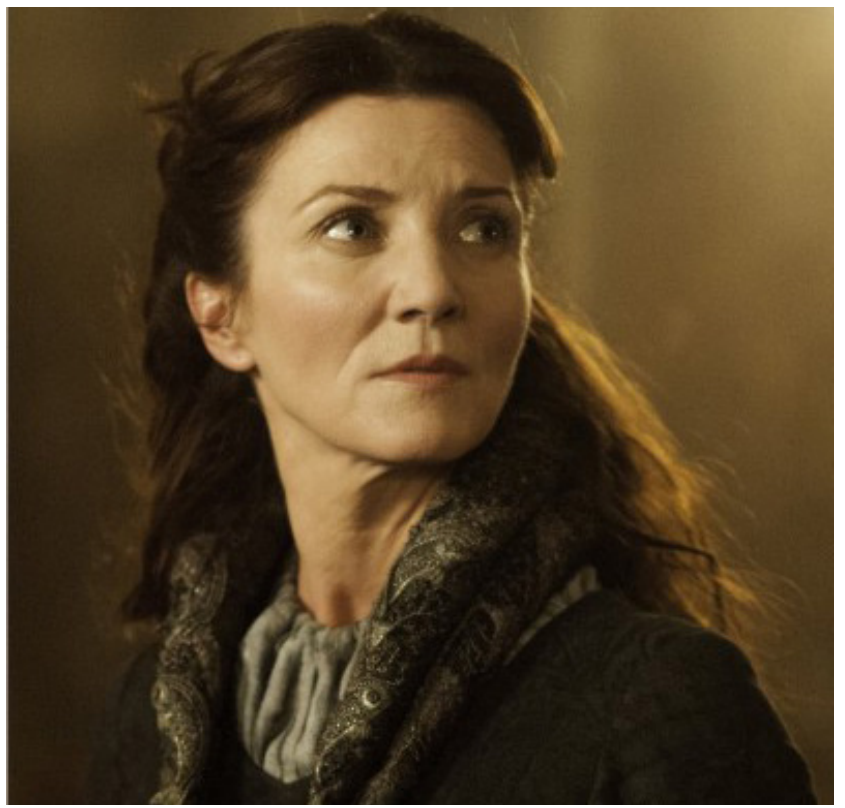

Llegamos de este modo a la figura, paradigmática en la antigüedad clásica, de la venganza femenina, a Medea, la verdadera mujer, para Lacan, vale decir, la que nos presenta en su acto la verdad del goce femenino, que trasciende así definitivamente a figura de la madre ideal.

"Medea es una de las más conocidas y fuertes tragedias de Eurípides, que aborda el tema de maltrato de una mujer y de su feroz venganza. Medea desposa al héroe Jasón, viven una vida feliz por algunos años en Corinto y tienen dos niños. Hasta que Jasón abandona a Medea y decide casarse con la princesa de Corinto” (Euripides, 2017)

Medea decide vengarse $y$, tras el tormento mental que la tragedia nos describe entre el amor a sus hijos y el odio al hombre que deja, lo castiga asesinando a los niños y a la amante de éste. Comete los crímenes y luego huye, en la carroza del dios del sol, Helios, privando a Jason incluso de la satisfacción de castigarla a ella misma, hasta ese punto llega su sed de venganza. Jacques - Alain Miller sitúa con precisión esta encrucijada del deseo femenino:

"En consecuencia, es una división del deseo la que, llevada al extremo, conduce al acto de Medea, ese acto que ilustra perfectamente, aunque de una forma que causa horror, que el amor materno no se basa sólo en la pura reverencia a la ley del deseo, o que se sostiene en ella únicamente a condición de que en la madre haya una mujer que siga siendo para un hombre la causa de su deseo. Así, pues, quizás cuando Jason se va, Medea deja de estar en esa posición” (Miller, 2005)

La venganza en la mujer puede ser ilimitada, como el propio goce femenino, cuando se siente despojada, o traicionada, por el amor de un hombre, del hombre que ocupó para ella ese lugar del Otro para el que ella se hizo causa de un deseo, al precio incluso de ser otra que sí misma en el propio acto que consuma dicha venganza.

Salvando las distancias, pues no se trataría de la misma estructura, es lo que vemos en el último capítulo de la sexta temporada, cuando a consecuencia del acto vengativo de Cersei, su hijo se ve arrastrado al abismo. Ella no deja de sonreír. Con Cersei no se trata de la pérdida del amor de un hombre, y de la pérdida por ende de su lugar de causa del deseo para éste, sino de algo de un orden mucho más narcisista.

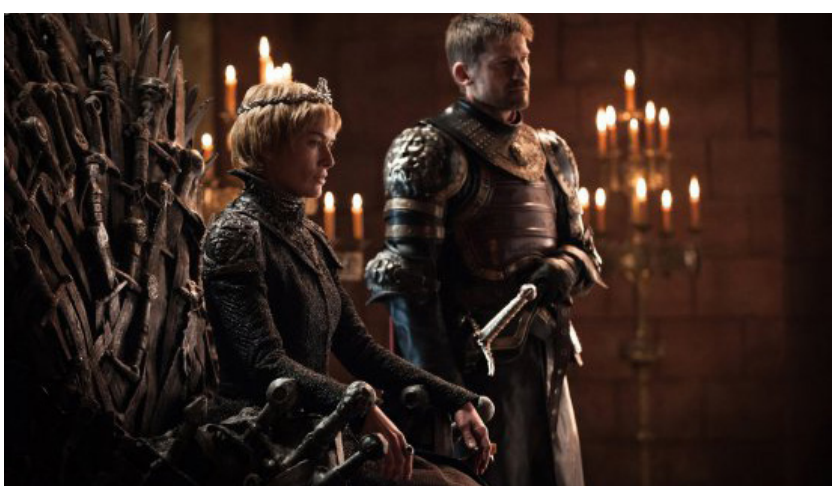


Entonces, para concluir, tres madres y un trono: Catelyn, consumida por odio y planeando la venganza por la muerte de sus hijos, queda reducida a ser un resto de piedra, vale decir, nada ubicamos en ella respecto a un deseo de ocupar el famoso trono. Daenerys tiene en su mito de nacimiento inscripta la muerte de su propia madre, y luego en su vida se topa con la brujería que signa la muerte del hijo que llevaba en su vientre, y la de su hombre. A partir de allí, en el acto simbólico del fuego del que emerge como madre guerrera, mantiene sus ansias de venganza, bajo el velo de ideales de justicia. Madre de dragones y de su pueblo, ella sí es una madre que ansía desde ese lugar el trono que le pertenece por derecho. Cersei, en cambio, sacrifica en la catedral del mundo a todos por una sed de venganza infinita, venganza descarnada y a cielo abierto, sin ningún velo, que resulta insoportable incluso para su propio hijo, que ante la revelación de ese cataclismo se arroja a ese vacío. Cersei ocupa actualmente ese trono, no como madre, eso queda claro, sino como una mujer con ansias infinitas de reivindicación y venganza.

\section{Referencias}

Brousse, M. H. (1993): “Femme ou mere?” en L’Autre sexe. La cause freudienne 24. Revue de psychanalyse. (Traducción propia).

Catelyn Tully, Wikipedia. Disponible en: https://es.wikipedia.org/wiki/Catelyn_Tully

Cersei Lannister, Wikipedia. Disponible en: https://es.wikipedia.org/wiki/Cersei_Lannister

Daenerys Targaryen, Wikipedia. Disponible en: https://es.wikipedia.org/wiki/Daenerys_Targaryen

Euripides, (2017): Encyclopædia Britannica. Ultimate Reference Suite. Chicago: Encyclopædia Britannica. (Traducción propia)

Freud, S. (1916 - 1938 [1945]): “La feminidad”. Nuevas lecciones introductorias al psicoanálisis, Obras completas, tomo III. Madrid: Biblioteca nueva.

Lacan, J. (2001 [2012]): “Nota sobre el niño”. Otros Escritos. Argentina: Paidos.

Miller, J. A. (2005): “El niño, entre la mujer y la madre” en Virtualia. Revista Digital de la Escuela de la Orientación Lacaniana, Junio / Julio 2005. Año IV, N 13. Disponible en: http://virtualia.eol.org.ar/013/default.asp?notas/miller.html 